\title{
MONOCHROMATIC IMAGES OF SUNSPOTS IN LINEARLY POLARIZED RADIATION AND STRUCTURE OF THEIR MAGNETIC FIELDS
}

\author{
YE SHI-HUI \\ Yunnan Astronomical Observatory, Academia Sinica \\ Kunming. 650011 \\ P.R. China \\ JIN JIE-HAI \\ Purple Mountain Observatory, Academia Sinica \\ Nanj̈̈n., 210008 \\ P.R. China
}

\begin{abstract}
The monochromatic images of unipolar sunspots in the Stokes parametcrs $Q$ and $U$ of magneto-sensitive lincs display a complicated structure. This is caused by the magneto-optical effect and also connected with the 3-D structure of spot magnetic fields. In the process of numcrical simulation it is possible to check the regularitics of the change of the anglc of inclination of magnetic lines of force with distance from spot center.
\end{abstract}

\section{INTRODUCTION}

The monochromatic $Q$ and $U$ images of unipolar sunspots cxhibit an interesting configuration. Each image consists of four quadrants and the neighboring ones have altcrnately plus and minus signs. Moreover, each quadrant contains a set of concentric closed curves. Figure 1 is the $U$ image of a large spot observed in FeI $\lambda 5324$ on April 28, 1988 at the Huairou Station of the Bcijing Observatory. Figure 2 is the longitudinal magnetogram of the same spot. It is impressive to note that these two images, belonging to one and the samc spot, have drastically different shapes. Observations of the Marshall Space Flight Center yicld the same result.

\section{THEORETICAL INTERPRETATION}

The peculiar configuration of the $Q$ and $U$ images of sunspots can be interpreted with the magneto-optical effect (Landi Degl' Innocenti, 1979). On the 


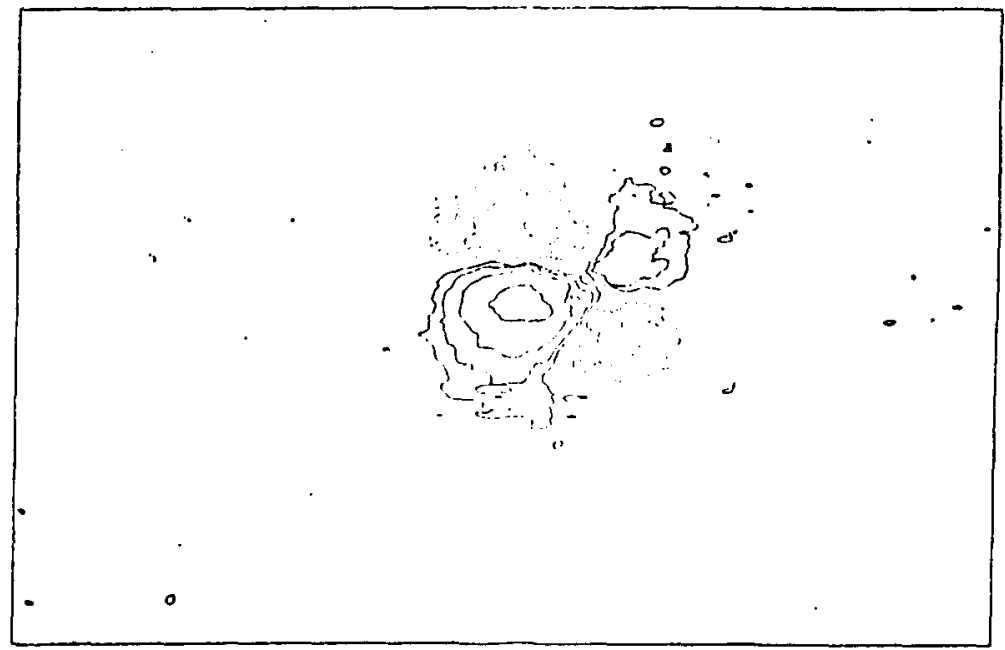

Fig. 1. Monochromatic $U$ image of a sunspot.

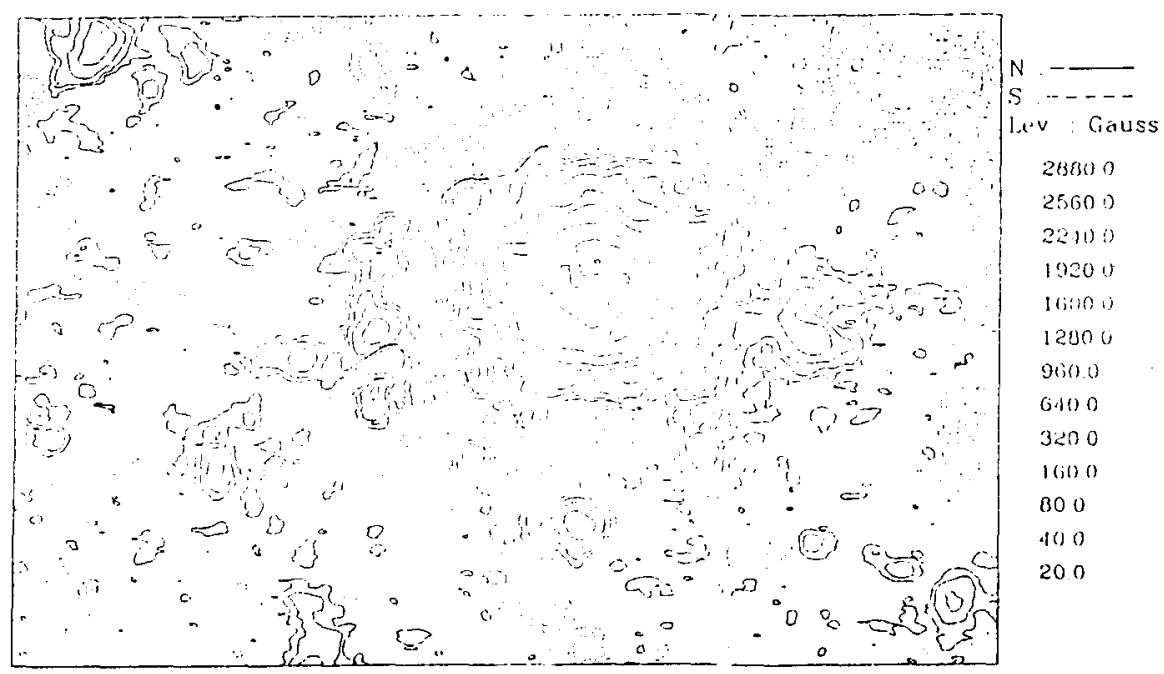

Fig. 2. Longitudinal magnetogram of the same spot.

basis of our former work (Ye Shi-hui and Jin Jie-bai, 1986), we performed numerical solutions of Unno-Beckers' equations, i.e.

$$
\left\{\begin{array}{l}
\cos \theta \frac{d I}{d \tau}=\left(1+\eta_{l}\right)(I-B)+\eta_{Q} Q+\eta_{U} U+\eta_{V} V, \\
\cos \theta \frac{d Q}{d \tau}=\eta_{Q}(I-B)+\left(1+\eta_{l}\right) Q+\rho_{\alpha} U+\rho_{\nu} \sin 2 \chi V, \\
\cos \theta \frac{d U}{d \tau}=\eta_{U}(I-B)-\rho_{a} Q+\left(I+\eta_{l}\right) U-\rho_{\nu} \cos 2 \chi V \\
\cos \theta \frac{d V}{d \tau}=\eta_{r}(I-B)-\rho_{\eta} \sin 2 \chi Q+\rho_{\nu} \cos 2 \chi U+\left(I+\eta_{l}\right) V .
\end{array}\right.
$$


The magneto-optical effect is represented by the following two coefficients:

$$
\left\{\begin{array}{l}
\rho_{R}=\frac{\eta_{0}}{H(0, a)} \cos \gamma\left[F\left(v-v_{H}, a\right)-F\left(v+v_{H}, a\right)\right], \\
\rho_{\nabla}=\frac{\eta_{0}}{H(0, a)} \frac{\sin ^{2} \gamma}{2}\left[F\left(v-v_{H}, a\right)+F\left(v+v_{H}, a\right)-2 F(v, a)\right],
\end{array}\right.
$$

where

$$
F(v, a)=\frac{1}{2 \pi} \int_{-\infty u^{2}+a^{2}}^{\infty} \frac{v}{-\left(u-u^{2}\right.} d u
$$

Moreover, we computed the signals of linear polarization defined by

$$
\begin{aligned}
& \Phi=\int 2(\lambda) f(\lambda) d \lambda \mid \int(x) f(\lambda) d i . \\
& \sigma=\int U(\lambda) \int(\lambda) d \partial \int f(\lambda) f(\lambda) d \lambda
\end{aligned}
$$

where $f(\lambda)$ is the transparency profile of the birefringent filter. Then we calculated for sunspots their theoretical $Q$ and $U$ images, which agree rather well with the observed ones.

\section{INCLINATION OF MAGNETIC LINES OF FORCE}

It is interesting to note that the theoretical $Q$ and $U$ spot images are concerned with the inclination angle $\gamma$ of magnetic lines of force to the normal on the solar surface. In the fan-shaped model (Hale and Nicholson, 1938) $\gamma$ is equal to a $\frac{\pi}{2} \rho$, where $a$ is an adjustable coefficient and $\rho$ is the distance from the spot center divided by the spot radius. We have found that for $a=1$ theoretical images agree well with observed ones. But when a becomes smaller, e.g. $a=0.833$ and 0.3 , the agreement is worse (see Figure 3 ). This may possibly mean that for the fan-shaped model we have to use the formula $\gamma=\frac{\pi}{2} \rho$.
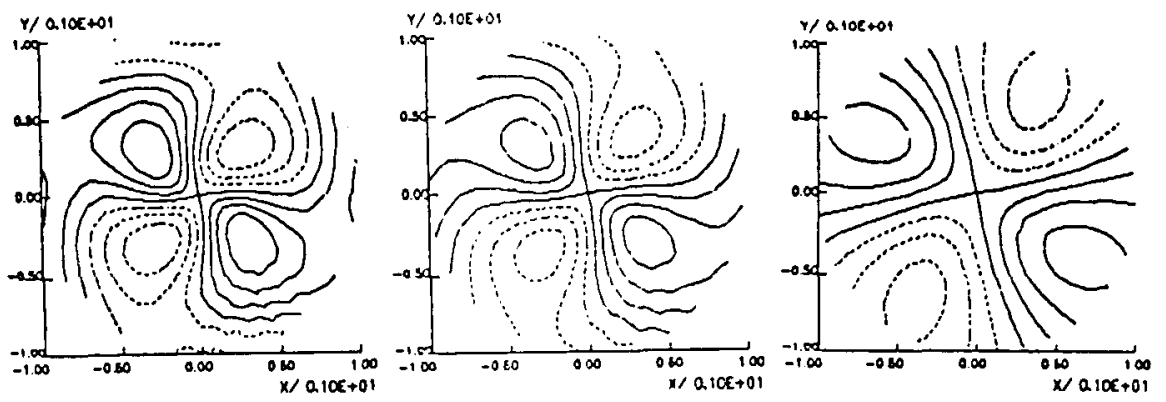

Fig. 3. Theoretical $Q$ images computed for fan-shaped model with $a=1,0.833$ and 0.3 (from left to right). 
We have also donc numcrical simulation with Osherovich's (1982) return-flux model and the agrecment betwecn theory and observation secms to be still better (figure 4). Therefore, we can say that this model may be closer to reality than the classical fan-shaped model.

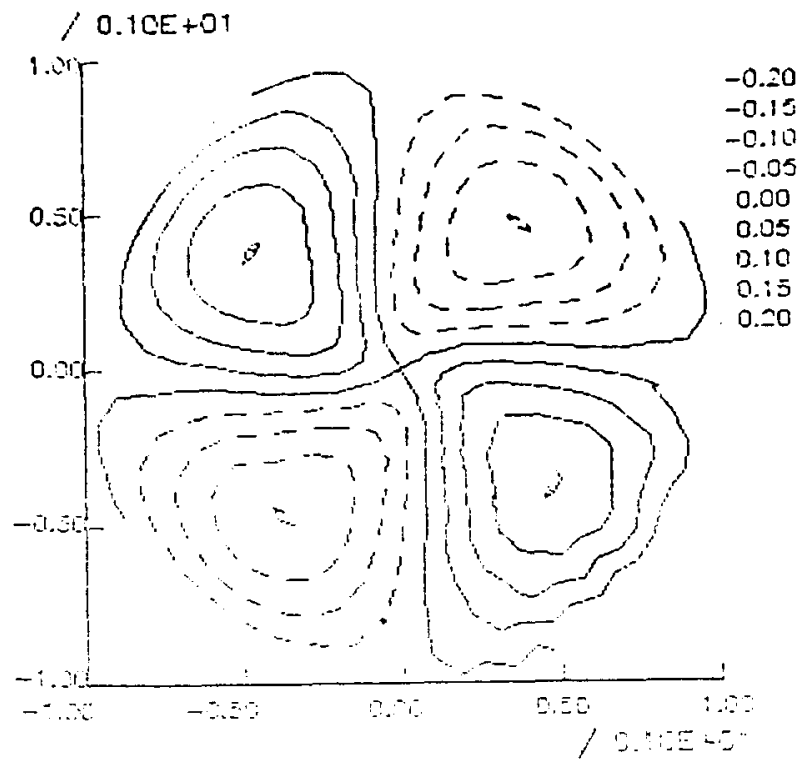

Fig. 4. Theoretical U image calculated with return-flux model.

\section{ACKNOWLEGEMENTS}

We would like to express our sincere gratitude to Drs. Ai Guoxiang and M. J. Hagyard for providing us precious observational data.

\section{REFERENCES}

Hale, G. E. and Nicholson, S. B. 1938, Magnetic Observations of Sunspots 1917--1924, Carnegie Institute of Washington.

Landi Degl'Innocenti, E. 1979, Solar Phys., 63, 237.

Osherovich, V. A. 1982, Solar Phys., 77, 63.

Ye Shi-hui and Jin Jie-hai 1986, Solar Phys., 104, 273. 